\title{
MicroRNA- 39 Suppresses the Tumorigenicity of Triple Negative Breast Cancer Cells by Targeting SOX8
}

\author{
Liangliang Dong' \\ Dongmei Zhou ${ }^{2}$ \\ Chunxia $\mathrm{Xin}^{3}$ \\ Bing Liu $^{3}$ \\ Ping Sun ${ }^{3}$
}

'Department of Medical Oncology, Yantai Yuhuangding Hospital, Cheeloo College of Medicine, Shandong University, Jinan, Shandong 250012, People's Republic of China; ${ }^{2}$ Department of Oncology, Yantai Shan Hospital, Yantai 264000, People's Republic of China; ${ }^{3}$ Department of Oncology, The Affiliated Yantai Yuhuangding Hospital of Qingdao University, Yantai 264000, People's Republic of China
Correspondence: Ping Sun

Department of Oncology, The Affiliated Yantai Yuhuangding Hospital of Qingdao

University, No. 20, Yuhuangding East

Road, Zhifu District, Yantai 264000,

People's Republic of China

Tel +86-13963896536

Email sunping20039@hotmail.com
This article was published in the following Dove Press journal: Cancer Management and Research

Purpose: The effects of miR-139 on the tumorigenicity of triple negative breast cancer (TNBC) and the underlying mechanisms were investigated.

Methods: Normal human breast epithelial (MCF-10A) and TNBC cell lines (HCC1806 and BT549) were used for microRNA (miR)-139 overexpression, SOX8 overexpression, and knockdown studies as in vitro models of TNBC. The expression of SOX8 and miR-139 was detected by reverse transcription-polymerase chain reaction. CCK 8 and clone formation assays were used to evaluate cell proliferation ability. Transwell assays and flow cytometry were used to test cell migration and apoptosis, respectively. Cell tumorigenicity was examined by tumor sphere formation assays. The interaction between miR-139 and SOX8 was examined by dual-luciferase reporter assays. The expression of SOX8, cleaved caspase- 3 , and cleaved caspase- 9 was analyzed by Western blotting. The findings were validated in vivo using a nude mouse transplanted tumor model.

Results: SOX8 expression was higher $(\mathrm{P}<0.05)$ and miR-139 expression was lower $(\mathrm{P}<$ 0.05 ) in HCC1806 and BT549 cells than in MCF-10A cells. SOX8 overexpression significantly enhanced cell proliferation and migration, reduced the rate of cell apoptosis, and increased tumor sphere formation $(\mathrm{P}<0.05)$ compared with the control group, whereas SOX8 knockdown had the opposite effect $(\mathrm{P}<0.05)$. Overexpression of miR-139 markedly decreased cell proliferation and migration, increased cell apoptosis in vitro, and decreased tumor angiogenesis and volume in vivo $(\mathrm{P}<0.05)$.

Conclusion: miR-139 suppressed the tumorigenicity of TNBC cells by targeting SOX8.

Keywords: breast cancer, carcinogenesis, molecular targets, gene regulation

\section{Introduction}

Breast cancer is one of the most common cancers in women worldwide and its incidence is increasing. ${ }^{1}$ Triple negative breast cancer (TNBC) is a form of breast cancer characterized by negative estrogen receptor (ER), progesterone receptor (PR), and human epidermal growth factor 2 (HER2) expression. It accounts for approximately $15-20 \%$ of all breast cancers, and is the most invasive and aggressive type of breast cancer. ${ }^{2}$ Because of limited treatment options, poor response to systemic therapy, and high aggressiveness and early widespread metastasis, the overall prognosis of patients with TNBC remains dismal. ${ }^{3}$ Moreover, the high degree of heterogeneity and the complexity of molecular mechanisms limit the development of novel therapeutic strategies. ${ }^{4,5}$ Currently, adjuvant chemotherapy for TNBC is based on standard chemotherapy regimens including anthracyclines, 
taxol, and cyclophosphamide. ${ }^{6}$ Investigating how to prevent, delay, or even suppress the rapid growth of TNBC has become a hot area of basic and clinical research in recent years.

MicroRNAs (miRNAs) are a group of small non-proteincoding RNA molecules of 20-22 nucleotides in length that can regulate gene expression by binding to specific target sites of mRNAs. They have been implicated in various diseases and are regarded as potential biomarkers or key regulators of numerous cellular processes. ${ }^{7,8}$ Many miRNAs are involved in the biological process of breast cancer. ${ }^{7}$ The dysregulation of miRNA expression is closely related to the mechanism of disease development, especially the occurrence of invasion and migration in breast cancer. 9,10 Accumulating evidence indicates that miR-139-5p may play a significant role in cancer biology, diagnosis, prognosis, and therapy. ${ }^{11}$ A previous study demonstrated that miR-139$5 \mathrm{p}$ may serve as a novel serum biomarker for colorectal cancer recurrence and metastasis. ${ }^{12}$ Watanabe et al reported that silencing miR-139 by histone methylation promotes non-small-cell lung cancer cell invasion. ${ }^{13}$ Pajic et al reported that miR-139 suppresses proliferation, migration, and invasion of tumor cells in epithelial ovarian cancer and in breast cancer. ${ }^{4}$ However, there have been few studies on the effects of miR-139 in TNBC.

In this study, we investigated the role of miR-139 in TNBC and the underlying molecular mechanisms in vitro and in vivo.

\section{Materials and Methods Cell Culture}

The normal human breast epithelial cell line MCF-10A and the TNBC cell lines HCC1806 and BT549 were purchased from Shanghai Institute of Biochemistry and Cell Biology affiliated with the Chinese Academy of Sciences (Shanghai, China). Cells were cultured in DMEM/F12 medium (Gibco, Invitrogen, Grand Island, NY, USA) supplemented with $1 \%$ penicillin/streptomycin and $10 \%$ fetal bovine serum in a constant temperature incubator (DRHA100, Shanghai, China) with $5 \% \mathrm{CO}_{2}$ at $37^{\circ} \mathrm{C}$. Cells were collected at the logarithmic growth phase when reaching approximately $80 \%$ confluence.

\section{Detection of Sry-Like High-Mobility Group Box (SOX8) mRNA Expression in Cells}

Cells were centrifuged at $4^{\circ} \mathrm{C}(12,000 \mathrm{rpm}, 15 \mathrm{~min})$ after harvesting and total RNA was extracted and isolated using the TRIzol Kit (Takara, Dalian, China). The OD260/ OD280 value (between 1.8 and 2.0) was used as an indicator of RNA purity. cDNA was transcribed using a reverse transcription kit (Roche, 11939823001, Shanghai, China). A Mastercycler $^{\circledR}$ nexus X2 (Eppendorf, Guangzhou, China) was used to perform reverse transcription-PCR (RT-PCR). The reaction conditions were as follows: $15 \mathrm{~s}$ at $95^{\circ} \mathrm{C}, 60 \mathrm{~s}$ at $60^{\circ} \mathrm{C}$, and 40 $\mathrm{s}$ at $72^{\circ} \mathrm{C}$ (35 cycles). The data were organized and analyzed using the $2^{-\Delta \Delta \mathrm{Ct}}$ method. The relative expression level was calculated after normalizing to GAPDH mRNA as the internal control. The sequences of the primers (Shenggong Bioengineering Technology Service Co., Ltd., Shanghai, China) used were the following.

miR-139: 5'-GCCTCTACAGTGCACGTGTCTC-3' (F), 5'-CGCTGTTCTCATCATCTGTCTCGC-3' (R);

U6: 5'-GACCTCTATGCCAACACAGT-3'(F), 5'-AGT ACTTGCGCTCAGGAGGA-3' (R);

SOX8: 5'-CGAGAGAAGACGCCTGCT-3' (F),

5'-CGTGTTGGAGAATGAGGG-3' (R);

GAPDH: 5'-TGACTTCAACAGCGACACCCA-3' (F), 5'-CACCCTGTTGCTGTAGCCAAA-3' (R).

\section{Dual-Luciferase Reporter Assay}

Wild-type (wt) and mutant (mut) 3' untranslated regions (3'-UTRs) of SOX8 were amplified in the pGL3/luciferase vector (Promega, E1751, Madison, WI, USA) and cloned downstream of the luciferase gene. The luciferase activities of HCC1806 and BT549 cells were detected using the dual luciferase reporter system following the manufacturer's instructions (Promega) at $48 \mathrm{~h}$ after transfection. ${ }^{14}$

\section{Cell Transfection}

Cells were cultured 1 day before transfection and inoculated into 6-well plates for lentivirus transfection. ${ }^{15}$ Full length SOX8 cDNA cloned into pcDNA3.1 mammalian expression plasmid (Shanghai GeneChem Co., Ltd, China). TNBC cells (HCC1806 and BT549) were divided into the following five groups according to treatment: 1) blank control group (BC group, no transfection), 2) SOX8 overexpression group (SOX8 group, cells were transfected with SOX8 pcDNA3.1 using Lipofectamine 3000), 3) SOX8 overexpression negative control group (NC1 group, cells transfected with SOX8 negative control), 4) SOX8 silencing group (si-S group, stable shSOX8 cells were constructed using short hairpin RNA (shRNA) with GeneChem), and 5) SOX8 silencing negative control group (NC2 group, cells transfected with shSOX8 negative 
control). RT-PCR and Western blotting were used to detect the transfection efficiency.

To investigate the effects of miR-139 on TNBC cells, the cells were grouped as follows: 1) blank control group (BC, no transfection), 2) miR-139 mimic group (miR-139, transfected with miR-139 mimic); and 3) and miR-139 analogue negative control group (miR-NC, transfected with unrelated mimic as negative control). The transfection efficiency and the expression of SOX8 in cells were detected by RT-PCR. miR-139 mimic and its negative control (NC), synthesized by Life Technologies Corporation, were transfected into cells using Lipofectamine 3000 reagent and the transfected cells were harvested at $72 \mathrm{~h}$ post-transfection.

To further explore the role of miR-139 in targeting SOX8, cells were divided into four groups as follows: 1) blank control group (BC), 2) miR-139 mimic group (miR139), 3) SOX8 silencing group (si-S), and 4) miR-139 mimics + SOX8 overexpression group $(\mathrm{miR}+\mathrm{S})$, in which cells were transfected with miR-139 mimic and SOX8 pcDNA3.1. Experiments were performed in triplicate for each group.

\section{Cell Proliferation Assay}

Transfected cells in the logarithmic growth stage were seeded into 96-well plates at a density of $2 \times 10^{4}$ cells/ $\mathrm{mL}$ with each well containing $100 \mu \mathrm{L}$. Cells were incubated in a $5 \% \mathrm{CO}_{2}$ incubator at $37^{\circ} \mathrm{C}$ for $24,48,72$, and $96 \mathrm{~h}$, and CCK-8 solution $(10 \mu \mathrm{L}$, Tongren Institute of Chemistry, Japan) was added to each well. The solution was mixed and cultured for $4 \mathrm{~h}$. The absorbance (OD) of each well was quantified by adjusting to the blank control as zero at $450 \mathrm{~nm}$.

\section{Plate Clone Formation Assay}

Cells were grown to the logarithmic growth phase, harvested, and digested with $0.25 \%$ trypsin to generate single cell suspensions. Cell density was adjusted to 250 cells/ $\mathrm{mL}$, and $2 \mathrm{~mL}$ of suspension was seeded into each well of a 6 -well plate. The plate was incubated at $37^{\circ} \mathrm{C}$ under $5 \%$ $\mathrm{CO}_{2}$ for 2-3 weeks, and the culture media were replaced every 3 days. Cells were then fixed with methanol, and $1 \mathrm{~mL}$ Giemsa working fluid (G1015, Solarbio, Beijing, China) was added to each well and incubated for $30 \mathrm{~min}$. Finally, the plate was washed with ultrapure water and photographed using a camera after the water around the plate was removed with filter paper.

\section{Cell Migration}

Pre-warmed DMEM medium ( $50 \mu \mathrm{L}$ per well) was evenly spread on the bottom of the upper chambers of a Transwell plate (3413, Corning, Beijing, China). Next, $100 \mu \mathrm{L}$ of cell suspension $\left(5 \times 10^{4}\right)$ was added to each well and incubated for $4 \mathrm{~h}$ at $37^{\circ} \mathrm{C}$. In the lower chamber, $600 \mu \mathrm{L}$ DMEM/F12 medium was added and cultured for $72 \mathrm{~h}$ in the same incubator. The chamber was then washed twice with PBS and fixed with $5 \%$ glutaraldehyde at $4{ }^{\circ} \mathrm{C}$. After two washes, the plates were stained with $0.1 \%$ Crystal Violet for $30 \mathrm{~min}$ and five fields $(400 \times)$ of view were randomly selected using an inverted microscope (Olympus, Japan) to calculate the mean value of migrated cells.

\section{Apoptosis Detection}

Cells treated as indicated were incubated for $24 \mathrm{~h}$, collected, resuspended in $1 \times$ precooled $\mathrm{PBS}\left(4^{\circ} \mathrm{C}\right)$, and centrifuged for 5-10 $\mathrm{min}$ at $1000 \mathrm{rpm} / \mathrm{min}$. After washing, cells were suspended with $1 \times$ binding buffer $(300 \mu \mathrm{L})$, and $5 \mu \mathrm{L}$ of Annexin V-APC (CA1020, Solarbio, Beijing, China) was added and mixed well. Cells were kept in the dark and incubated for $15 \mathrm{~min}$ at room temperature. Propidium iodide $(5 \mu \mathrm{L})$ was added 5 min before loading, followed by the addition of $1 \times$ binding buffer $(200 \mu \mathrm{L})$. Finally, flow cytometry (Beckman Coulter, Brea, CA, USA) was used to analyze samples, and the data were analyzed using CellQuest software (BD Bioscience, San Diego, CA, USA).

\section{Western Blotting Analysis}

Protein lysates were obtained from cells using a protein extraction kit (W037, Nanjing Jiancheng Bioengineering Institute, Nanjing, China). Total proteins (30 $\mu \mathrm{g})$ were subjected to $10 \%$ SDS-PAGE electrophoretic separation and the resolved proteins were transferred onto a polyvinylidene difluoride (PVDF) membrane (W0161-1, Nanjing Jiancheng Bioengineering Institute). Following blocking with non-fat milk powder for $2 \mathrm{~h}$, membranes were incubated with rabbit anti-human SOX8 (1:1000, ab226983, Abcam, Bejing, China), rabbit antihuman cleaved caspase-3 (1:4000, ab49822, Abcam), rabbit anti-human cleaved caspase-9 (1:20,000, ab2324, Abcam), rabbit anti-human $\beta$-actin (1:20,000, ab2324, Abcam) at $4^{\circ} \mathrm{C}$ for $12 \mathrm{~h}$. Subsequently, membranes were incubated with a goat anti-rabbit HRP-conjugated IgG H\&L (1:20,000, ab205718, Abcam) secondary antibody for $50 \mathrm{~min}$. The protein bands were visualized using an 
ECL chemiluminescence kit (32132, ThermoFisher, Shanghai, China) and $\beta$-actin was selected as a control to calculate the relative expression of the target proteins.

\section{Tumor Sphere Formation Assays}

DMEM/F12 serum-free medium (containing 1\% penicillin-streptomycin, $20 \mathrm{ng} / \mathrm{mL}$ EGF, $20 \mathrm{ng} / \mathrm{mL} \mathrm{bFGF}$, and $2 \% \mathrm{~B} 27$ ) was used to resuspend the cells, and the concentration was adjusted to $1 \times 10^{4} / \mathrm{mL}$. Cells were inoculated in a low adhesion 6-well plate at a density of 5000 cells per well, and the solution was changed every other day. After 7 days of culture, tumor spheres were collected when they reached a diameter of $50 \mu \mathrm{m}$, digested, dispersed into single cells, and reinoculated to form new tumor spheres. After 1 week, the number of tumor spheres with a diameter $>50 \mu \mathrm{m}$ was counted under an inverted microscope. The expression of miR139 in cells was detected by RT-PCR following RNA extraction of tumor spheres.

\section{In vivo Experiment Using a Murine TNBC Model}

Specific pathogen-free grade BALB/C female nude mice $(\mathrm{n}=48)$ (4-weeks-old, 16-18 g body weight) were obtained from the Beijing Vital River Laboratory Animal Technology Co., Ltd. [SCXK (Jing) 20190006, Beijing, China] and maintained in a sterile cage in an air laminar flow purifying room. The room was kept under constant temperature $\left(26-28^{\circ} \mathrm{C}\right)$ and humidity $(40-60 \%)$, and the feed, drinking water, and bedding were sterilized.

The 48 mice were randomly divided into a blank control group (BC), miR-139 mimic group (miR-139), SOX8 silencing group (si-SOX8), miR-139 mimic and SOX8 pcDNA3.1 group $(\mathrm{miR}+\mathrm{S})$, with 12 mice in each group. TNBC HCC1806 and BT549 cells in the logarithmic growth stage were digested with $0.25 \%$ trypsin, collected, and counted. The cell concentration was adjusted to $5 \times$ $10^{7} / \mathrm{mL}$, and the cell suspension $(0.1 \mathrm{~mL})$ was injected into the soft part of the skin on the back of the right forelimb of nude mice.

The tumor volume was calculated by measuring tumor length (L) and short diameter (W) using a Vernier caliper every 7 days as follows: $(\mathrm{V})=$ (long diameter $\times$ short diameter $\left.{ }^{2}\right) / 2$. After 28 days, mice were intraperitoneally anesthetized with $0.6 \%$ pentobarbital sodium $(40 \mathrm{mg} / \mathrm{kg})$ and euthanized. The tumor tissue was extracted and tumors were weighed.

\section{Statistical Analyses}

Analysis was performed using SPSS, version 19.0 (IBM, Chicago, IL, USA), and results are presented as the mean \pm standard deviation (SD). The differences among multiple groups were calculated by one-way analysis of variance (ANOVA) and Dunnett's $t$-test. A value of $\mathrm{P}<0.05$ was considered statistically significant.

A

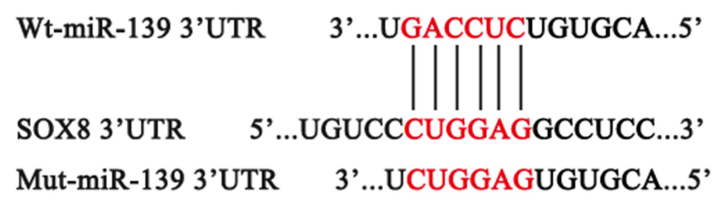

B

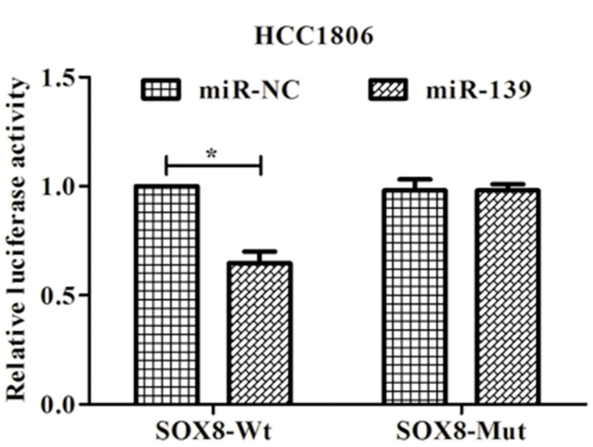

C

BT549

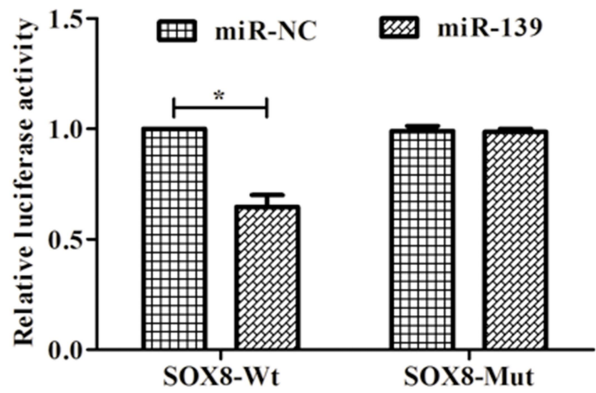

Figure I SOX8 is a target gene of miR-I39 in TNBC cells. (A) The predicted binding sites of miR-I39 and the SOX8 $3^{\prime}-\mathrm{UTR}$ region according to TargetScan. (B, C) Luciferase activity in cells following co-transfection with miR-139 mimic and luciferase reporters containing wild type (wt)- or mutated (mut)-SOX8 transcript in HCCI806 cells and BT549 cells. *P $<0.05$. 


\section{Results}

miR-I39 Bound to the 3'UTR of SOX8 in

\section{Dual Luciferase Reporter Assay}

SOX8 was predicted as a candidate target of miR-139 using

TargetScan (http://www.targetscan.org) (Figure 1A). A dualluciferase reporter assay was performed to confirm that miR139 targeted SOX8 in HCC1806 (Figure 1B) and BT549 cell lines (Figure 1C). The results showed that when miR-139 mimic was co-transfected with wild-type SOX8 3'-UTR, the luciferase activity decreased ( $\mathrm{P}<0.05$ ), while a reversal of luciferase expression was observed in experiments where miR-139 mimic was co-transfected with mut-SOX8.

\section{SOX8 Promoted the Proliferation of TNBC Cells}

As shown in Figure 2A and B, the expression of SOX8 mRNA and protein was significantly higher in HCC1806 and BT549 cells than in MCF-10A cells (P $<0.05$ ). Differences in expression of SOX8 in HCC1806 and BT549 cells were observed by RTPCR. In the SOX8 group, the expression of SOX8 was significantly increased compared with the control group ( $\mathrm{P}<0.05$, Figure $2 \mathrm{C}$ and $\mathrm{D}$ ), and the expression of SOX8 was clearly decreased in the si-SOX8-treated group compared with the control group $(\mathrm{P}<0.05$,

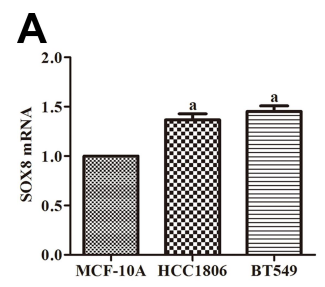

B
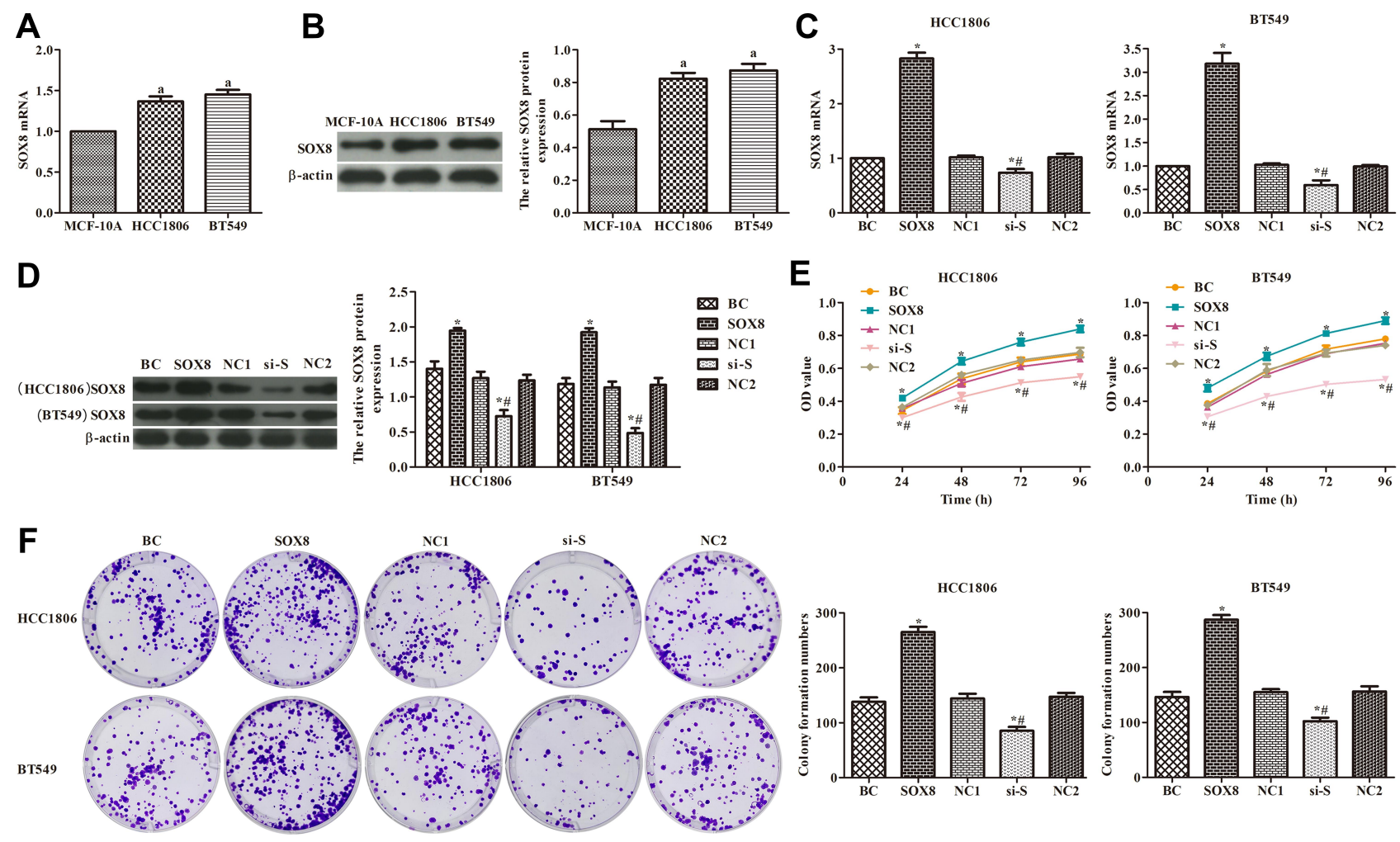

\section{$E$}

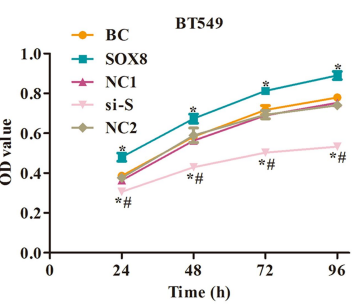

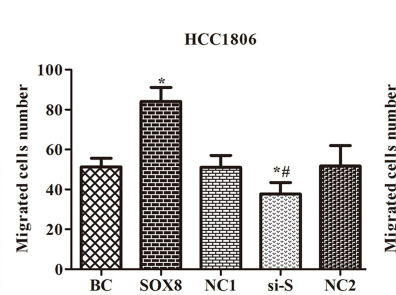

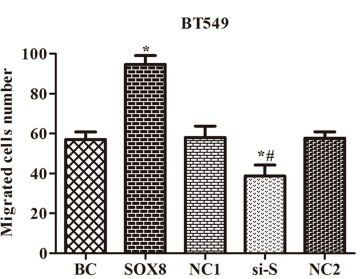

Figure 2 Effect of SOX8 on the proliferation of TNBC cells. (A and B) Expression of SOX8 mRNA in cells measured by RT-PCR and Western blotting analysis. (C and D) Transfection efficiency of SOX8 evaluated by RT-PCR and Western blotting analysis. (E) Cell viability detected by the CCK8 assay. (F) Cell proliferation was measured by plate clone formation. (G) Cell migration ability detected by transwell assay (400x). In each group, triplicate experiments were performed. The differences among groups were analyzed by ANOVA followed by the Dunnett's $t$-test. Compared with MCF-IOA cells, ${ }^{\mathrm{a}} \mathrm{P}<0.05$; compared with the BC group, $* \mathrm{P}<0.05$; compared with the SOX8 group, ${ }^{\#} \mathrm{P}<0.05$.

Abbreviations: BC, blank control group; SOX8, SOX8 overexpression group; $\mathrm{NCI}$, SOX8 overexpression negative control group; si-S, SOX8 silencing group; NC2, SOX8 silencing negative control group. 
Figure 2C and D). In order to analyze the effects of SOX8 on the proliferation of TNBC cells, cell proliferation was measured by CCK-8 expression (Figure $2 \mathrm{E}$ ), plate clone formation assays (Figure $2 \mathrm{~F}$ ), and the transwell migration assay (Figure 2G). The results showed that SOX8 overexpression notably increased the TNBC cell viability and migration, and silencing SOX8 expression significantly decreased the TNBC cell viability and migration, when compared with control cells $(\mathrm{P}<0.05)$. These data suggested that higher expression of SOX8 in TNBC cells promoted cell proliferation.
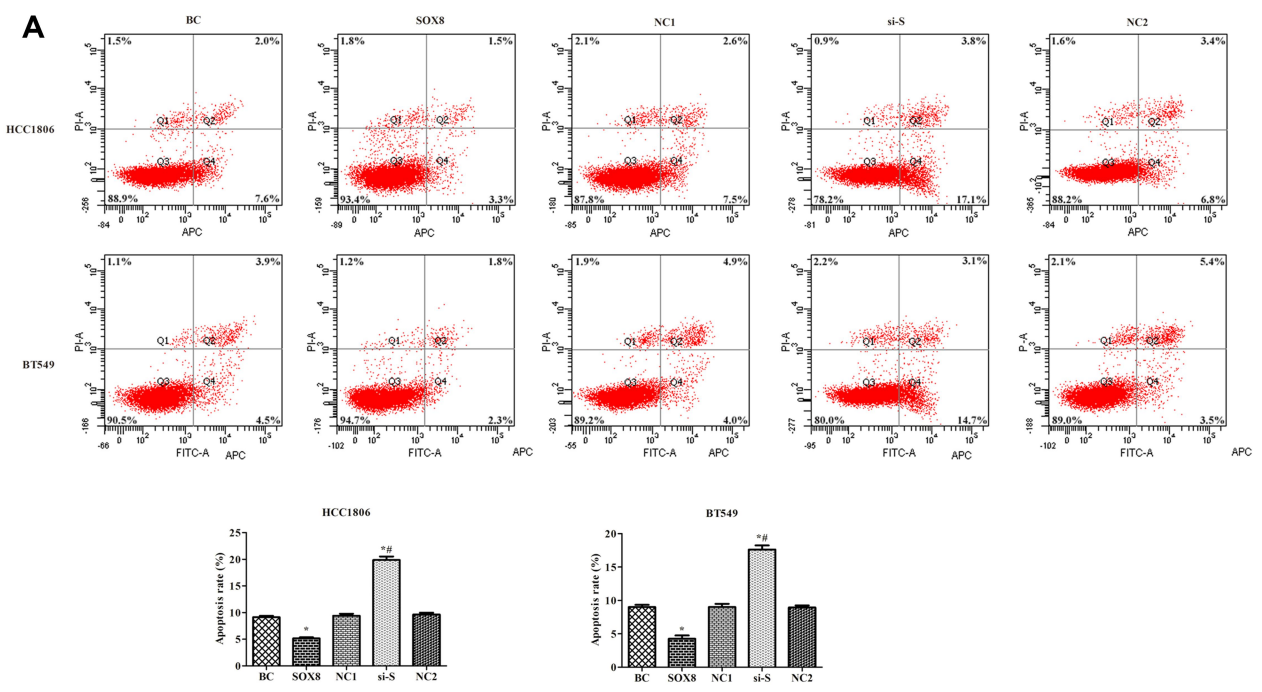

B
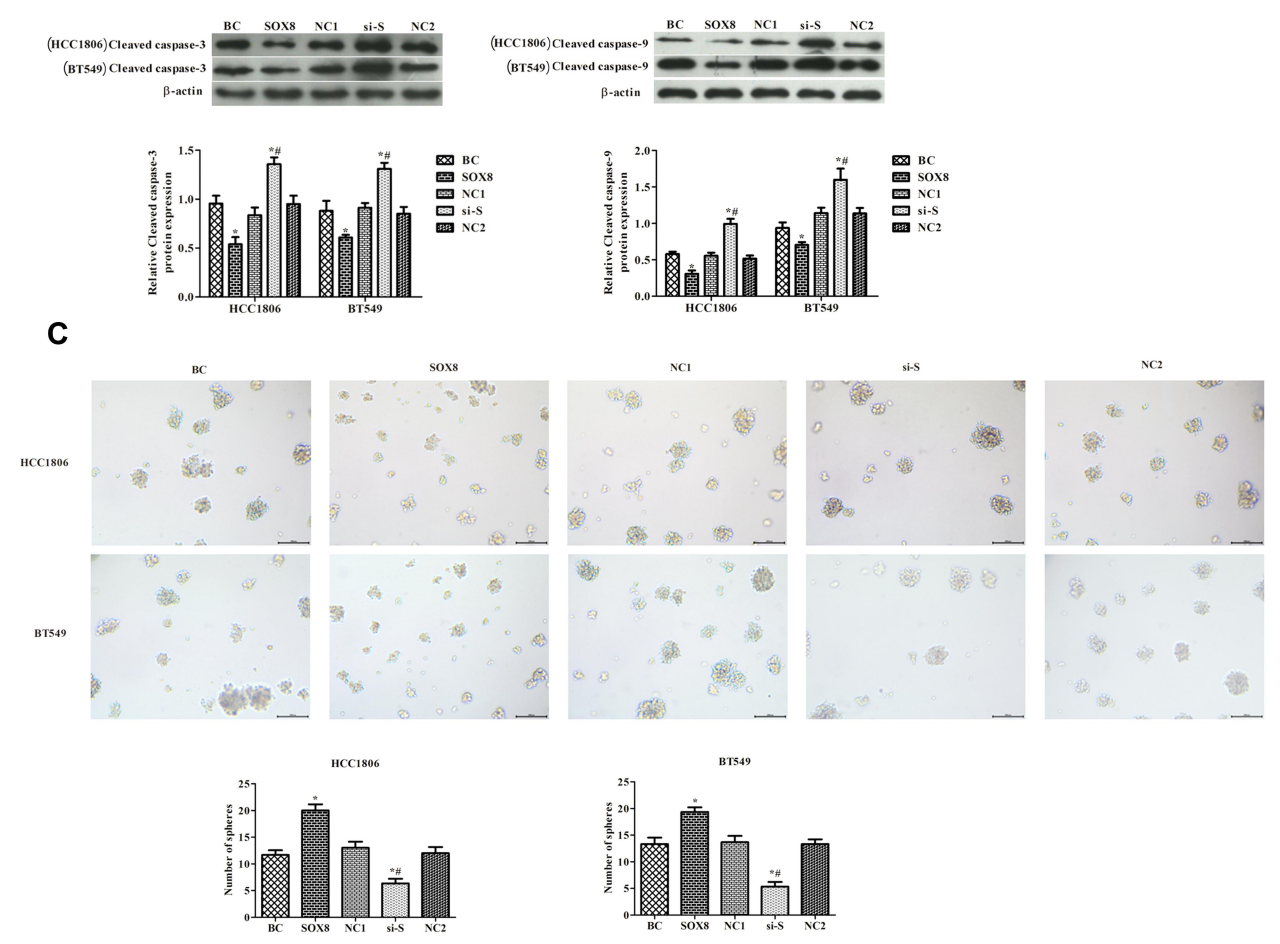

Figure 3 Effects of SOX8 on TNBC cell apoptosis and tumor sphere formation. (A) Apoptosis was detected by flow cytometry. (B) The expression of cleaved caspase-3 and cleaved caspase- 9 was analyzed by Western blotting analysis. (C) Tumorigenicity of cells was determined by in vitro tumor sphere formation assays (I00x). In each group, experiments were performed in triplicate. The differences among groups were analyzed by ANOVA followed by the Dunnett's $t$-test. Compared with the BC group, ${ }^{*} \mathrm{P}<0.05$; compared with the SOX8 group, ${ }^{\#} \mathrm{P}<0.05$.

Abbreviations: BC, blank control group; SOX8, SOX8 overexpression group; NCI, SOX8 overexpression negative control group; si-S, SOX8 silencing group; NC2, SOX8 silencing negative control group. 


\section{SOX8 Overexpression Inhibited}

Apoptosis of TNBC Cells and Promoted Tumor Sphere Formation

The effects of SOX8 expression on TNBC cell apoptosis were analyzed by flow cytometry (Figure $3 \mathrm{~A}$ ). The apoptosis rate was markedly decreased in the SOX8 group and increased in the si-SOX8-treated group, when compared with the control group ( $\mathrm{P}<0.05)$. The expression of cleaved caspase- 3 and cleaved caspase- 9 was observed using Western blotting analysis (Figure 3B). Consistent with the results of the apoptosis rate, the levels of cleaved caspase- 3 and cleaved caspase- 9 were significantly increased in the si-S group and decreased in the SOX8 group, compared with the control group $(\mathrm{P}<0.05)$. The formation of tumor spheres of HCC1806 and BT549 cells was also observed (Figure 3C). Compared with the control group, the number of tumor spheres was significantly higher in the SOX8 group overexpressing SOX8, while the number of tumor spheres was significantly lower in the si-S group $(\mathrm{P}<$ 0.05). These results indicated that higher expression of SOX8 in TNBC cells inhibited cell apoptosis and promoted tumor sphere formation in vitro.

\section{Overexpression of miR-I39 Inhibited the Proliferation of TNBC Cells}

As shown in Figure 4A, the expression of miR-139 was remarkably lower in HCC1806 and BT549 cells than in MCF-10A cells $(\mathrm{P}<0.05)$, indicating that miR-139 was downregulated in TNBC cells. In order to evaluate the effects of miR-139 on the proliferation of TNBC cells, the overexpression of miR-139 was induced in HCC1806 and BT549 cells by transfecting miR-139 mimic (Figure $4 \mathrm{~B})$. We found that the expression of SOX8 was lower in
A

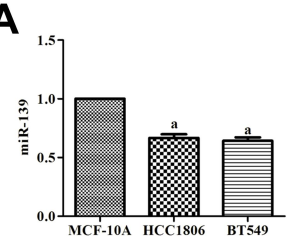

D
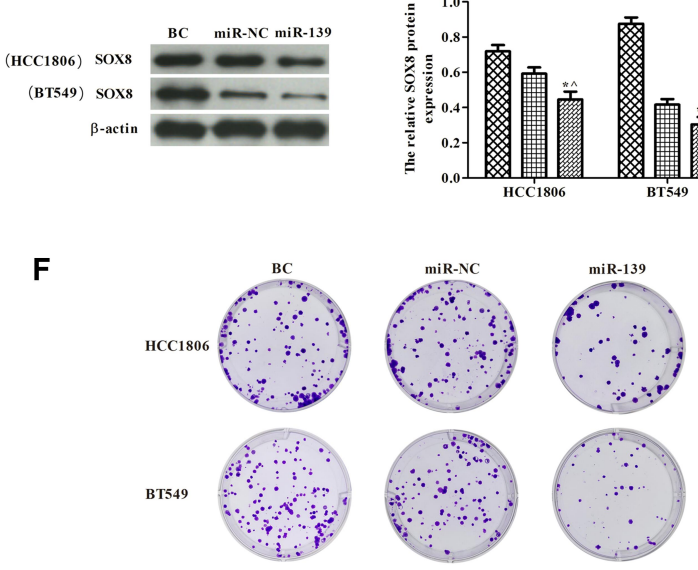

G
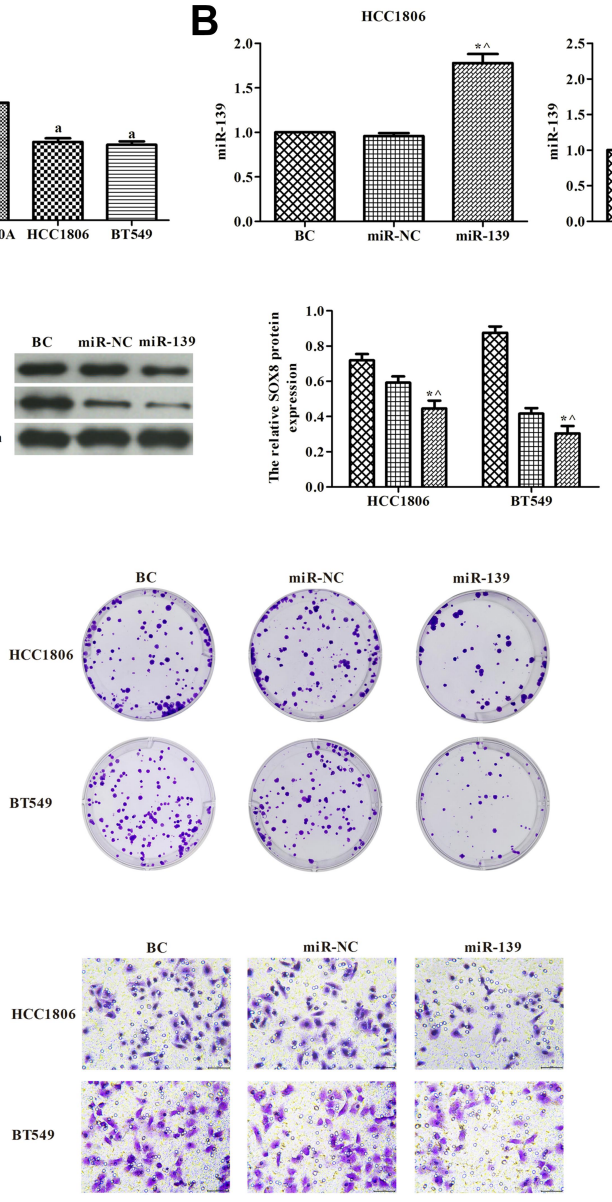
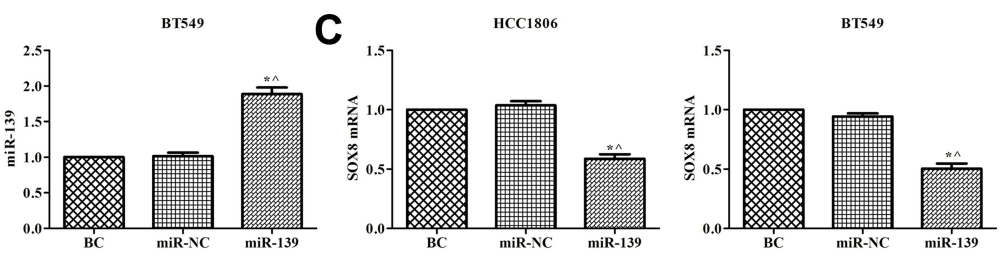

E
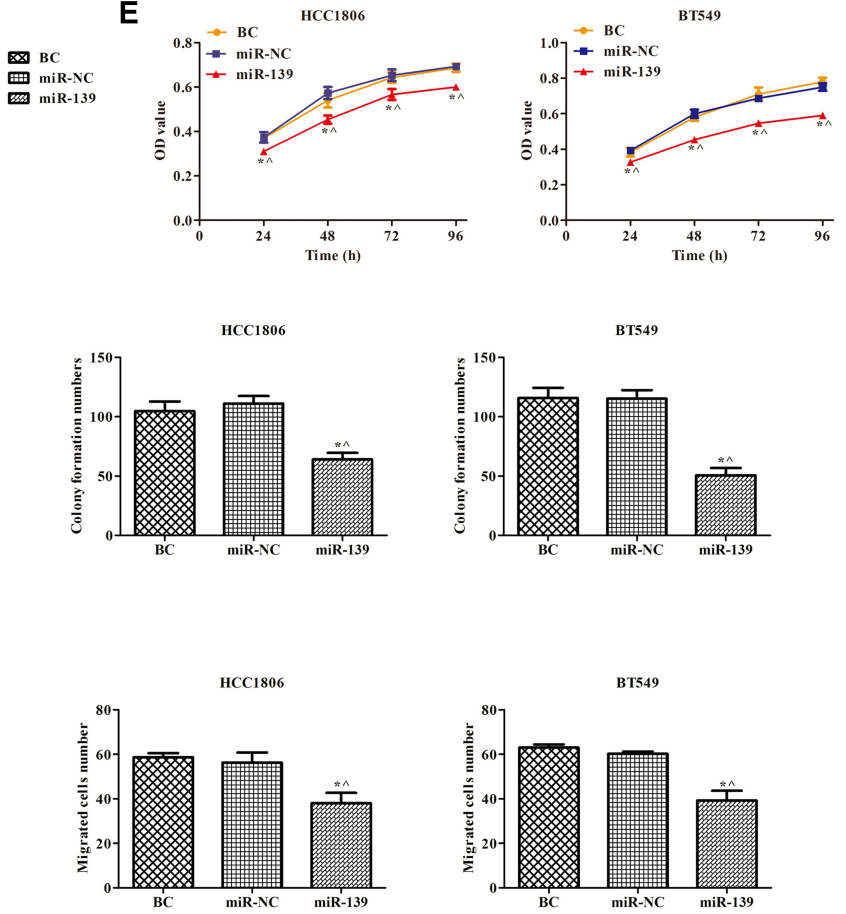

Figure 4 Effects of miR-I39 on the proliferation of TNBC cells. (A) RT-PCR was used to detect the expression of miR-I39 in cells. (B) Transfection efficiency of miR-I39 evaluated by RT-PCR. (C and D) RT-PCR and Western blotting analysis were used to detect the expression of SOX8 in cells. (E) Cell viability detected by CCK8 assay. (F) Cell proliferation was measured by plate clone formation. (G) Transwell assays were used to detect cell migration ability (400x). In each group, experiments were performed in triplicate. The differences among groups were analyzed by ANOVA with followed by the Dunnett's $t$-test. Compared with MCF-10A cells, ${ }^{\text {a }} \mathrm{P}<0.05$; compared with the $\mathrm{BC}$ group, $* \mathrm{P}<0.05$; compared with the miR-NC group, ${ }^{\wedge} \mathrm{P}<0.05$.

Abbreviations: BC, blank control group; miR-NC, miR-I39 analogue negative control group; miR-I39, miR-139 mimics group. 
cells overexpressing miR-139 compared with the control group (Figure $4 \mathrm{C}$ and $\mathrm{D}, \mathrm{P}<0.05$ ). The proliferation of TNBC cells was tested by measuring CCK-8 (Figure 4E), the plate clone formation assay (Figure 4F), and transwell migration assay (Figure 4G). High expression of miR-139 significantly inhibited the viability of TNBC cells and their migration when compared with control cells $(\mathrm{P}<0.05)$. These data suggested that miR-139 overexpression inhibited the proliferation of TNBC cells.

\section{miR-I39 Induced Apoptosis of TNBC Cells and Inhibited Tumor Sphere Formation}

The effects of miR-139 expression on TNBC cell apoptosis were analyzed by flow cytometry (Figure 5A). The apoptosis rate was markedly higher in the miR-139 group compared with the control group $(\mathrm{P}<0.05)$. The expression of both cleaved caspase-3 and caspase-9 was observed by Western blotting (Figure 5B). Consistent

A
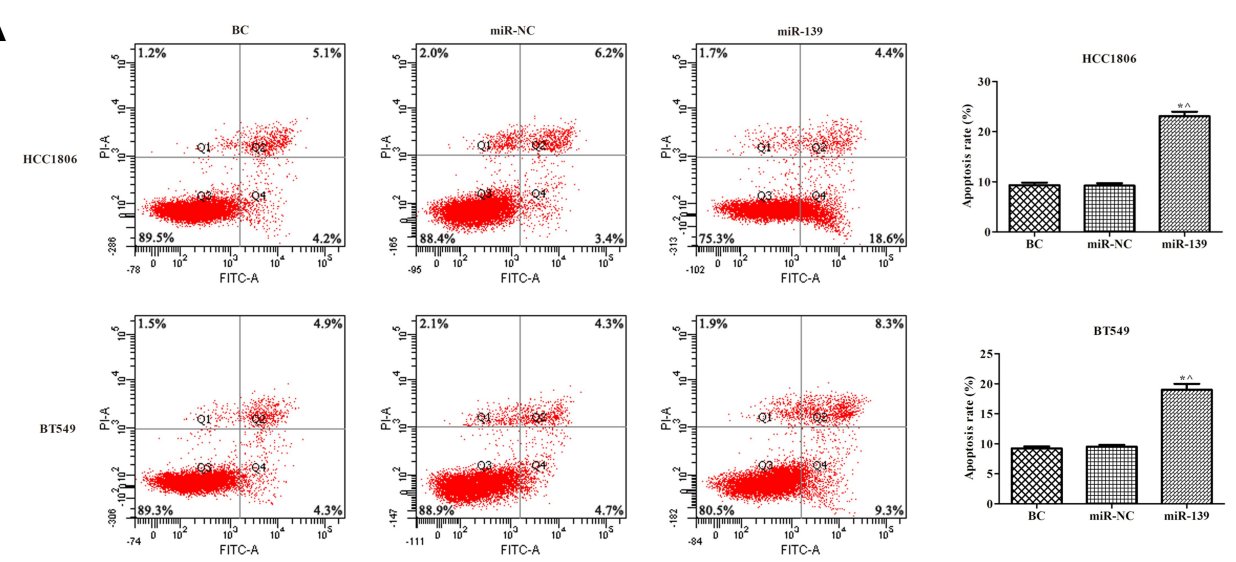

B
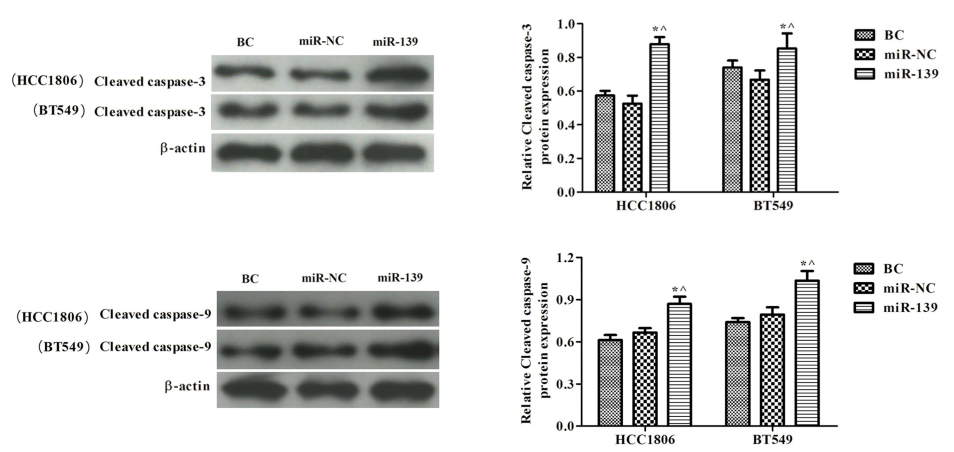

C
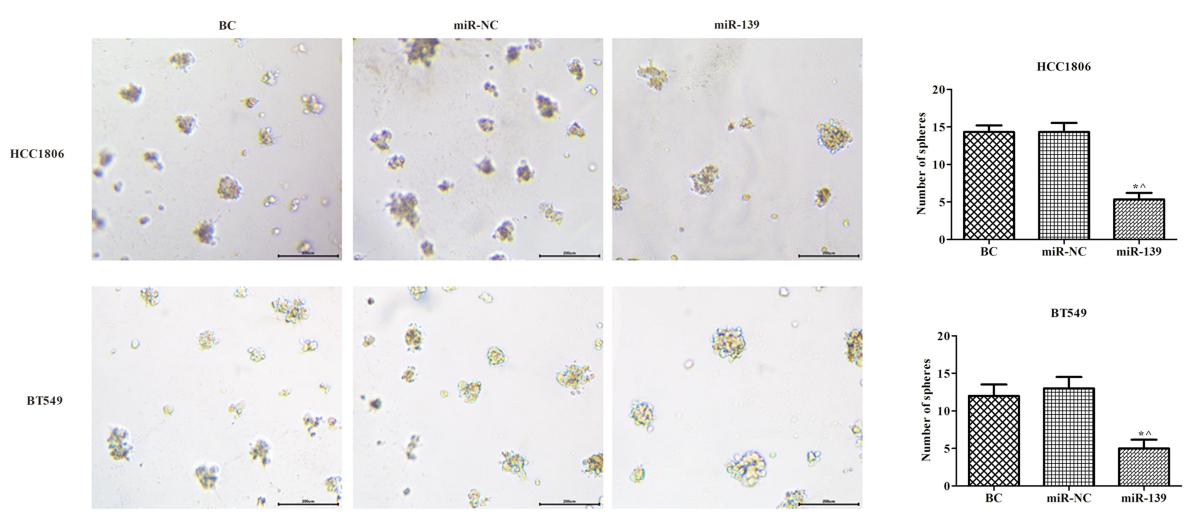

Figure 5 Effects of miR-139 on TNBC cell apoptosis and tumor sphere formation. (A) Cell apoptosis was detected by flow cytometry. (B) The expression of cleaved caspase- 3 and cleaved caspase- 9 was analyzed by Western blotting analysis. (C) Tumorigenicity of cells was determined by in vitro tumor sphere formation assays (I00x). In each group, experiments were performed in triplicate. The differences among groups were analyzed by ANOVA followed by the Dunnett's $t$-test. Compared with the BC group, *P $<0.05$; compared with the miR-NC group, $\wedge \mathrm{P}<0.05$.

Abbreviations: BC, blank control group; miR-NC, miR-139 negative control group; miR-139, miR-139 mimics group. 
with the results of the apoptosis rate, the levels of cleaved caspases were significantly increased in the miR-139 group, compared with the control group ( $\mathrm{P}<$ 0.05). The tumor sphere formation of $\mathrm{HCC} 1806$ and BT549 cells was also observed (Figure 5C). Compared with the control group, the number of tumor spheres was significantly lower in cells overexpressing miR-139 ( $\mathrm{P}<$ $0.05)$. These results revealed that higher expression of miR-139 in TNBC cells promoted cell apoptosis and restrained tumor sphere formation in vitro.

\section{miR- I39 Inhibited the Proliferation of TNBC Cells by Targeting SOX8}

We further investigated whether miR-139 regulated the proliferation of TNBC cells via targeting SOX8 expression. Overexpression of miR-139 and SOX8 was established in TNBC cells. The proliferation of TNBC cells was analyzed by measuring CCK-8 (Figure 6A), the plate clone formation assay (Figure 6B), and by transwell migration assay (Figure $6 \mathrm{C})$. The results showed that high expression of miR-139 inhibited the proliferation of TNBC cells, and high expression of SOX8 countered the effect of miR-139 overexpression $(\mathrm{P}<0.05)$. These data revealed that miR-139 suppressed proliferation of TNBC cells via regulating SOX8.

\section{miR-I39 Induced Apoptosis of TNBC Cells and Inhibited Tumor Formation by Targeting SOX8}

The apoptosis rate of TNBC cells was observed to determine whether miR-139 induced apoptosis via regulating SOX8. As shown in Figure 7A, the apoptosis rate was higher in the $\mathrm{miR}+\mathrm{S}$ group compared with the miR-139 group $(\mathrm{P}<0.05)$. Similarly, the levels of cleaved caspase- 3 and cleaved caspase- 9 were clearly decreased in the miR $+\mathrm{S}$ group compared with the miR139 group $(\mathrm{P}<0.05$, Figure $7 \mathrm{~B})$. Further, the tumor sphere formation of TNBC cells was significantly higher in the miR+S group than in the miR-139 group $(\mathrm{P}<$ 0.05 , Figure $7 \mathrm{C}$ ). In vivo, tumor formation was consistent with the results observed in vitro (Figure 7D). The tumor volume and weight were significantly higher in the $\mathrm{miR}+\mathrm{S}$ group than those in the miR-139 group $(\mathrm{P}<$ 0.05). Overall, these data suggested that miR-139 suppressed the tumorigenicity of TNBC cells by targeting SOX8.
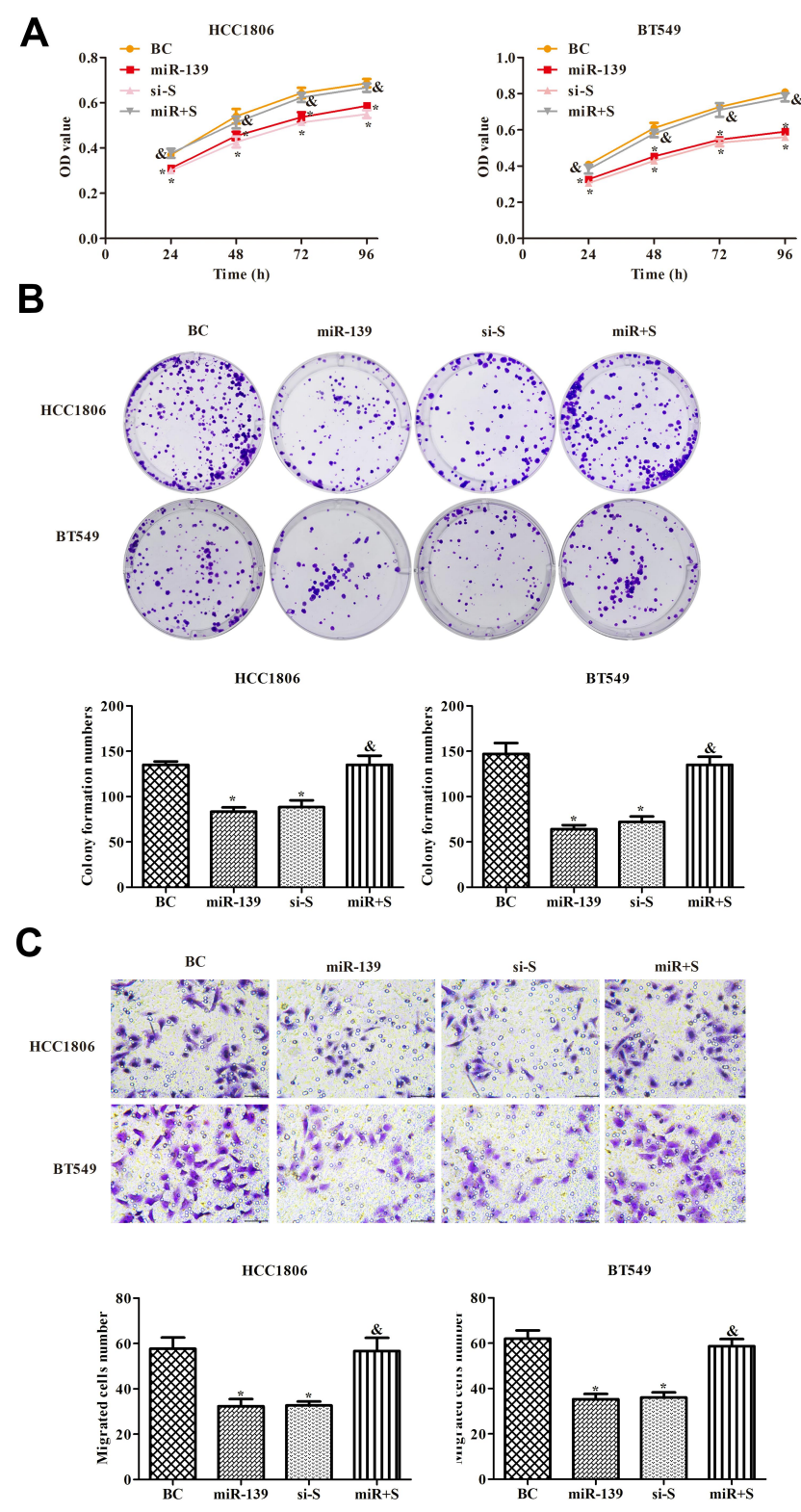

Figure 6 Effects of miR-139 on the proliferation of TNBC cells by targeting SOX8. (A) CCK8 was used to detect cell viability. (B) Plate clone formation experiment was used to detect cell proliferation. (C) Transwell assay was used to detect cell migration (400x). In each group, experiments were performed in triplicate. The differences among groups were analyzed by ANOVA followed by the Dunnett's $t$-test. Compared with the BC group, *P $<0.05$; compared with the miR- 139 group, \& $\mathrm{P}<0.05$.

Abbreviations: BC, blank control group; miR-139, miR-139 mimics group; si-S, SOX8 silencing group; miR+S, miR-139 mimics + SOX8 overexpression group.

\section{Discussion}

In previous studies, miR-139 has been found to be associated with aggressive tumor behavior and disease progression in breast cancer. MiR-139 expression was thus identified as a surrogate marker for tumor aggressiveness in breast cancer and was found to be downregulated in tumor tissues compared with controls. ${ }^{16-18}$ 
A
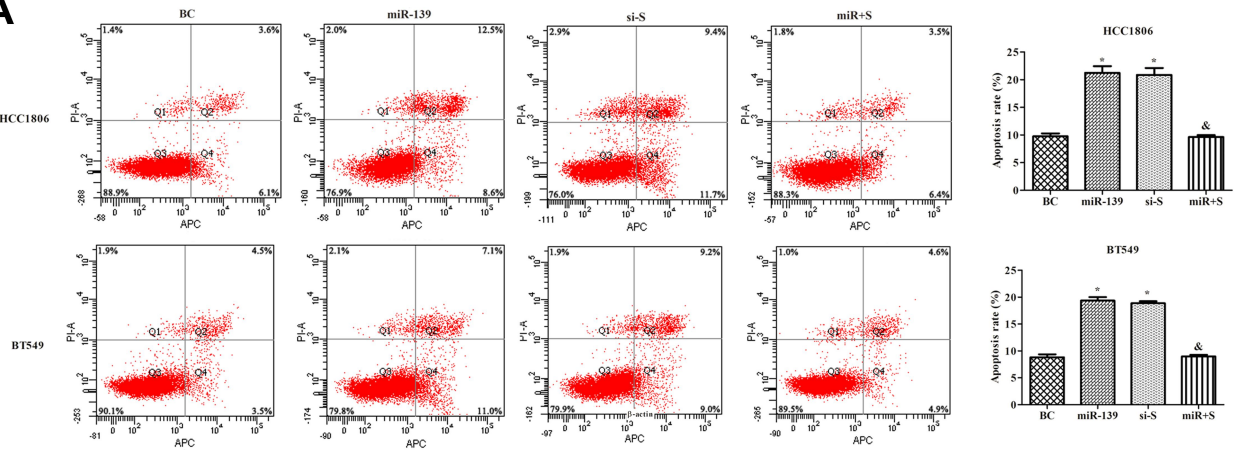

B
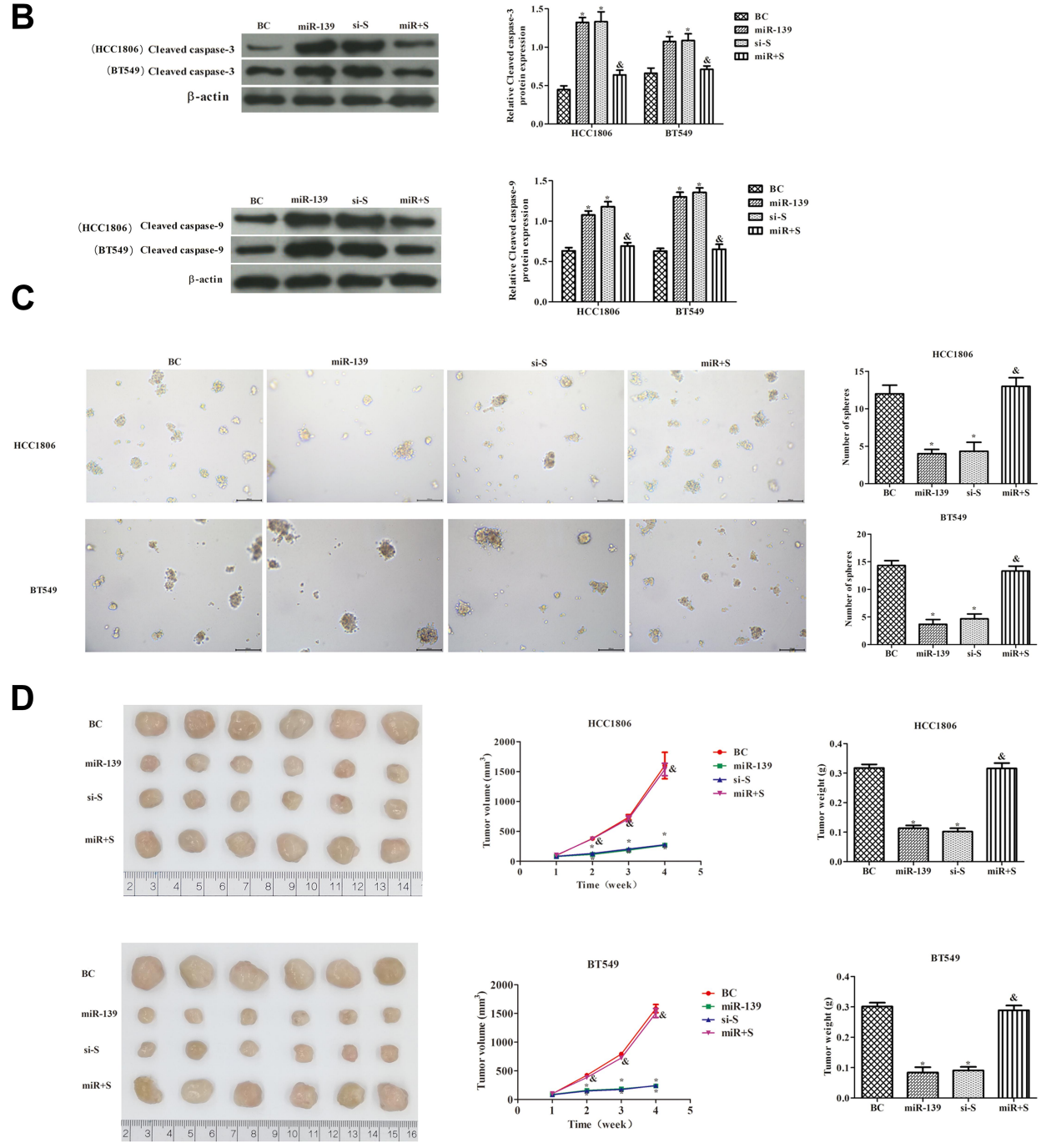

Figure 7 Effects of miR-139 targeting SOX8 on TNBC cell apoptosis and tumor formation. (A) Apoptosis of HCCI806 and BT549 cells measured by flow cytometry. (B) The expression of cleaved caspase- 3 and cleaved caspase- 9 was analyzed by Western blotting analysis. (C) In vitro, tumor sphere formation assays were used to detect the tumorigenicity of $\mathrm{HCCl} 806$ and BT549 cells (I00x). (D) In vivo, tumor volume and tumor weight of TNBC cells. In each group, there were six mice. The differences among groups were analyzed by ANOVA followed by Dunnett's $t$-test. Compared with the BC group, $* \mathrm{P}<0.05$; compared with the mir- 139 group, ${ }^{\&} \mathrm{P}<0.05$.

Abbreviations: BC, blank control group; miR-139, miR-139 mimics group; si-S, SOX8 silencing group; miR+S, miR-I39 mimics + SOX8 overexpression group.

SOX8 as a functional oncogene that is involved in the maintenance of stem-like capacities in TNBC cells, and its high expression has been significantly associated with poor prognosis in TNBC patients. ${ }^{19,20}$ However, whether miR-139 modulates TNBC cells and the underlying molecular mechanisms remain unclear. In this study, the dual-luciferase reporter assay was performed to confirm that miR-139 targets SOX8 in HCC1806 and 
BT549 cells. Further, we observed higher expression of SOX8 and lower expression of miR-139 in HCC1806 and BT549 cells, when compared with the normal human breast epithelial cell line MCF-10A. These findings support that miR-139 is a potential anti-metastatic miRNA and SOX8 is a potential pro-metastatic gene.

SOX genes have been well studied in many cancers, and have been described as oncogenes or tumor suppressors involved in tumor formation and progression. ${ }^{21}$ As a member of the SOXE subfamily, SOX8 is an important functional oncogene that promotes tumor growth in tongue squamous cell carcinoma, with poor prognosis. ${ }^{21,22}$ In the present study, SOX8 overexpression enhanced the proliferation of TNBC and decreased TNBC cell apoptosis. The expression levels of cleaved caspase- 3 and cleaved caspase-9 were also evaluated, which were decreased after SOX8 overexpression. When the expression of SOX8 was inhibited, the proliferation of TNBC was suppressed and apoptosis of TNBC increased.

A number of studies have reported that miR-139 plays an important role in the tumor aggressiveness of breast cancer, and is poorly expressed in such tumors. ${ }^{16,23}$ In breast cancer, cells treated with a miR-139-5p mimic strongly synergized with radiation exposure, resulting in significantly increased oxidative stress, accumulation of unrepaired DNA damage, and induction of apoptosis. ${ }^{4}$ The present results indicated that miR-139 may be a novel target for the treatment of breast cancer. Consistent with previous studies, our study found that miR-139 was down-regulated in TNBC cells. In addition, overexpression of miR-139 suppressed cell proliferation and promoted apoptosis of TNBC. In order to modify miR-139-inhibited tumor formation via targeting SOX8 in TNBC, cancer cells were transfected with miR-139 mimic and SOX8 pcDNA3.1, simultaneously. The results indicated that the effects of miR-139 were weakened by overexpression of SOX8.

Nonetheless, the mechanisms involved in miR-139 regulation of SOX8 in TNBC are not completely understood. For example, the downstream genes of SOX8 need to be explored in TNBC and effective targeted drugs need to be identified in future studies.

\section{Conclusions}

In summary, this study demonstrated that miR-139 inhibited the tumorigenicity of TNBC cells by targeting SOX8, providing a rationale for the clinical diagnosis and development of targeted therapeutic drugs in TNBC.

\section{Abbreviations}

TNBC, triple negative breast cancer; ER, estrogen receptor; PR, progesterone receptor; HER2, human epidermal growth factor 2; PVDF, polyvinylidene difluoride; SOX8, Sry-like high-mobility group box.

\section{Statement of Ethics}

All experimental protocols were performed according to the National Institutes of Health (NIH Pub. No. 85-23, revised 1996), and the experiments were approved by the Institutional Animal Care and Use Committee of Shandong University (No. 20190703-002).

\section{Author Contributions}

All authors made substantial contributions to conception and design, acquisition of data, or analysis and interpretation of data; took part in drafting the article or revising it critically for important intellectual content; agreed to submit to the current journal; and gave final approval of the version to be published. All authors agreed to be accountable for all aspects of the work.

\section{Funding}

There is no funding to report.

\section{Disclosure}

The authors have no conflicts of interest to declare for this work.

\section{References}

1. LaBarge MA, Samson S, Miyano M. Breast cancer beyond the age of mutation. Gerontology. 2015;62(4):434-442. doi:10.1159/000441030

2. Pogoda K, Niwinska A, Murawska M, Pienkowski T. Analysis of pattern, time and risk factors influencing recurrence in triple-negative breast cancer patients. Med Oncol. 2013;30(1):388. doi:10.1007/ s12032-012-0388-4

3. Cleator S, Heller W, Coombes RC. Triple-negative breast cancer: therapeutic options. Lancet Oncol. 2007;8(3):235-244. doi:10.1016/ S1470-2045(07)70074-8

4. Pajic M, Froio D, Daly S, et al. miR-139-5p modulates radiotherapy resistance in breast cancer by repressing multiple gene networks of DNA repair and ROS defense. Cancer Res. 2018;78(2):501-515. doi: 10.1158/0008-5472.CAN-16-3105

5. Liu D, Huo X, Gao L, Zhang J, Ni H, Cao L. NF-kappaB and Nrf2 pathways contribute to the protective effect of Licochalcone $\mathrm{A}$ on dextran sulphate sodium-induced ulcerative colitis in mice. Biomed Pharmacother. 2018;102:922-929. doi:10.1016/j.biopha.2018.03.130

6. Joerger M, Thurlimann B. Chemotherapy regimens in early breast cancer: major controversies and future outlook. Expert Rev Anticancer Ther. 2013;13:165-178. doi:10.1586/era.12.172

7. Bartel DP. MicroRNAs: genomics, biogenesis, mechanism, and function. Cell. 2004;116(2):281. doi:10.1016/S0092-8674(04)00045-5 
8. Shukla GC, Singh J, Barik S. MicroRNAs: processing, maturation, target recognition and regulatory functions. Mol Cell Pharmacol. 2011;3(3):83-92.

9. Zhong S, Li W, Chen Z, Xu J, Zhao J. MiR-222 and miR-29a contribute to the drug-resistance of breast cancer cells. Gene. 2013;531(1):8-14. doi:10.1016/j.gene.2013.08.062

10. Le Quesne J, Caldas C. Micro-RNAs and breast cancer. Mol Oncol. 2010;4(3):230-241. doi:10.1016/j.molonc.2010.04.009

11. Huang LL, Huang LW, Wang L, Tong BD, Ding XS. Potential role of miR-139-5p in cancer diagnosis, prognosis and therapy (Review). Oncol Lett. 2017;14(2):1215-1222. doi:10.3892/ol.2017.6351

12. Miyoshi J, Toden S, Yoshida K, et al. MiR-139-5p as a novel serum biomarker for recurrence and metastasis in colorectal cancer. Sci Rep. 2017;7:43393. doi:10.1038/srep43393

13. Watanabe K, Amano Y, Ishikawa R, et al. Histone methylation-mediated silencing of miR-139 enhances invasion of non-small-cell lung cancer. Cancer Med. 2015;4(10):1573-1582. doi:10.1002/cam4.505

14. Yan G, Li Y, Zhan L, et al. Decreased miR-124-3p promoted breast cancer proliferation and metastasis by targeting MGAT5. Am $J$ Cancer Res. 2019;9(3):585-596.

15. Feng X, Luo Q, Wang H, Zhang H, Chen F. MicroRNA-22 suppresses cell proliferation, migration and invasion in oral squamous cell carcinoma by targeting NLRP3. J Cell Physiol. 2018;233 (9):6705-6713. doi:10.1002/jcp.26331

16. Dai H, Gallagher D, Schmitt S, et al. Role of miR-139 as a surrogate marker for tumor aggression in breast cancer. Hum Pathol. 2017;61:68-77. doi:10.1016/j.humpath.2016.11.001
17. Hua W, Sa KD, Zhang X, et al. MicroRNA-139 suppresses proliferation in luminal type breast cancer cells by targeting Topoisomerase II alpha. Biochem Biophys Res Commun. 2015;463(4):1077-1083. doi:10.1016/j.bbrc.2015.06.061

18. Gu SQ, Luo JH, Yao WX. The regulation of miR-139-5p on the biological characteristics of breast cancer cells by targeting COL11A1. Math Biosci Eng. 2019;17(2):1428-1441. doi:10.3934/ mbe. 2020073

19. Tang H, Chen B, Liu P, et al. SOX8 acts as a prognostic factor and mediator to regulate the progression of triple-negative breast cancer. Carcinogenesis. 2019;40(10):1278-1287. doi:10.1093/carcin/bgz034

20. Chen B, Tang H, Chen X, et al. Transcriptomic analyses identify key differentially expressed genes and clinical outcomes between triple-negative and non-triple-negative breast cancer. Cancer Manag Res. 2018;11:179-190. doi:10.2147/CMAR.S187151

21. Xie SL, Fan S, Zhang SY, et al. SOX8 regulates cancer stem-like properties and cisplatin-induced EMT in tongue squamous cell carcinoma by acting on the $\mathrm{Wnt} / \beta$-catenin pathway. Int $J$ Cancer. 2018;142(6):1252-1265. doi:10.1002/ijc.31134

22. Chen S, Li H, Li X, et al. High SOX8 expression promotes tumor growth and predicts poor prognosis through GOLPH3 signaling in tongue squamous cell carcinoma. Cancer Med. 2020;9 (12):4274-4289. doi:10.1002/cam4.3041

23. Rask L, Balslev E, Søkilde R, et al. Differential expression of miR-139, miR-486 and miR-21 in breast cancer patients sub-classified according to lymph node status. Cell Oncol (Dordr). 2014;37(3):215-227. doi:10.1007/s13402-014-0176-6

\section{Publish your work in this journal}

Cancer Management and Research is an international, peer-reviewed open access journal focusing on cancer research and the optimal use of preventative and integrated treatment interventions to achieve improved outcomes, enhanced survival and quality of life for the cancer patient.
The manuscript management system is completely online and includes a very quick and fair peer-review system, which is all easy to use. Visit http://www.dovepress.com/testimonials.php to read real quotes from published authors. 